\title{
Descentralización de los servicios de salud: estudios de caso en seis estados mexicanos
}

\author{
Nuria Homedes, PhD, (1) Antonio Ugalde, PhD. (2)
}

\begin{abstract}
Homedes N, Ugalde A.
Descentralización de los servicios de salud: estudios de caso en seis estados mexicanos. Salud Publica Mex 20I 1;53:493-503.
\end{abstract}

\begin{abstract}
Resumen
Objetivo. Este artículo presenta los resultados de la segunda descentralización de la Secretaría de Salud de México (1994-2000). Material y métodos. Además de revisar documentos oficiales y de archivo, estadísticas de salud y de productividad, se realizaron observaciones en los centros asistenciales y 232 entrevistas de profundidad en los estados de Baja California Sur, Colima, Guanajuato, Nuevo León, Sonora y Tamaulipas con directivos de las secretarías de salud estatales y jurisdiccionales, dirigentes de sindicatos, prestadores de servicios y representantes de la sociedad civil. Resultados. Se identifican los problemas que los estados tuvieron que enfrentar para implementar la descentralización. Conclusiones. No se alcanzaron los objetivos trazados por los promotores de la descentralización.
\end{abstract}

Palabras clave: descentralización; salud; reforma; México
Homedes N, Ugalde A.
Decentralization of health services:
Case studies in six Mexican states.
Salud Publica Mex 20 I I;53:493-503.

\section{Abstract}

Objective. This article discusses the effects of the second decentralization of the Mexican Ministry of Health (19942000). Material and Methods. It is based on a review of official and archival documents, health and productivity statistics, observations in clinics and hospitals, and 232 indepth interviews in the states of Baja California Sur, Colima, Guanajuato, Nuevo León, Sonora and Tamaulipas. The interviewees included high-level administrators of state and district health systems, leaders of workers unions, health providers and representatives of civil society. Results. The article identifies the problems that offices of health at state level had to overcome to implement the decentralization. Conclusions. Descentralizacion failed to achieve the objectives stated by its promoters.

Key words: decentralization; health; reform; Mexico
$\mathrm{D}$ urante las últimas décadas, como consecuencia de diversas presiones políticas y económicas, ${ }^{1,2}$ la mayoría de los países latinoamericanos efectuaron reformas en el sector salud encaminadas a su descentralización y privatización. La crisis económica que empezó en la década de los ochenta originó que los gobiernos de México y otros países de medianos y bajos ingresos no pudieran pagar su deuda externa. El Banco Mundial y el Fondo Monetario Internacional (FMI) presionaron a los gobiernos a fin de que realiza- ran ajustes estructurales que exigían la reducción del gasto en educación, salud y otros servicios sociales ${ }^{3}$ a través de reformas sectoriales; de no hacerlo, estas dos instituciones no estaban dispuestas a conceder más préstamos. Posteriormente otros organismos financieros y agencias bilaterales apoyaron estas propuestas. La descentralización permitiría a los gobiernos centrales pasar la carga financiera de los servicios sociales a otras subdivisiones administrativas, ya fuesen estados, regiones, provincias o municipios.

(I) School of Public Health, University of Texas-Houston. Houston, Texas, EUA

(2) Department of Sociology, University of Texas-Austin. Austin, Texas, EUA 
En 1983, México fue uno de los primeros países en implementar la descentralización en respuesta a la severa recesión económica que atravesaba el país.

En este trabajo se distinguen dos etapas en el proceso de implementación: la primera corresponde al periodo presidencial 1982-1988; y la segunda se refiere a las administraciones de 1988 a 1994 y de 1994 a 2000 . La administración 1988-1994 no interrumpió oficialmente la descentralización pero de facto se recentralizaron los servicios sociales.

Durante el sexenio de 1994-2000 se avanzó en la descentralización. El gobierno en turno durante 20002006 no se manifestó en contra de ésta, pero desde el principio empezó a diseñar el Sistema de Protección Social en Salud, conocido como Seguro Popular (SP), destinado a la población no cubierta por la seguridad social. El SP fue diseñado y es financiado por la federación; lo implementan los estados y fue prioritario para esa administración y la siguiente (2006-2012). El SP condiciona la transferencia de los fondos federales al número de familias que se inscriben en el programa, y por lo tanto representa una recentralización del poder decisorio. ${ }^{4}$

La primera etapa de la descentralización tuvo poco éxito ${ }^{5-8^{*}}$ y su costo fue elevado: 452 millones de dólares. ${ }^{9}$ No transfirió poder decisorio y los fondos seguían adjudicándose desde el centro. Sólo 14 de los 31 estados aceptaron descentralizarse. ${ }^{5,10}$ Los gobernadores que se negaron intuyeron que la descentralización transferiría más responsabilidades sanitarias que recursos y poder decisorio. Durante 1988-1994 hubo una contrarreforma, centralizándose en la oficina presidencial el manejo de grandes fondos para programas sociales. ${ }^{11,12}$

En México se han publicado estudios cuantitativos de la segunda descentralización de varios estados. Un estudio incluyó los estados de Guanajuato, San Luis Potosí y Zacatecas. ${ }^{13}$ Un segundo estudio hizo un análisis de los presupuestos en cuatro estados (Hidalgo, Oaxaca, Tabasco y Yucatán) e incluyó algunas encuestas de profundidad. ${ }^{14}$ Otro estudio valioso de la segunda descentralización ${ }^{15}$ incluye datos cuantitativos, entrevistas cerradas en varios estados y entrevistas abiertas en tres (Aguascalientes, Guanajuato y San Luis Potosí).

En América Latina se ha avanzado mucho en la aplicación de las técnicas cualitativas a los estudios del sector salud. ${ }^{15,16}$ Los estudios cualitativos enriquecen el entendimiento de una realidad social que es imposible captar en estudios cuantitativos.

\footnotetext{
* Cardozo-Brum M. La política de descentralización de servicios de salud: análisis de su proceso y evaluación de resultados. México DF, (Documento no publicado), 1995.
}

\section{Objetivos}

El objetivo del presente artículo es comparar los resultados de los estudios cualitativos que examinan la segunda etapa de la descentralización mexicana (1994-2000) con las metas anticipadas por las agencias mencionadas anteriormente: ${ }^{17-20}$ fortalecer la participación comunitaria y la democracia, mejorar la eficiencia y mejorar la calidad de la atención (cuadro I).

Los estudios de caso que cumplen con estos criterios de inclusión se realizaron en los estados de Baja California Sur, Colima, Guanajuato, Nuevo León, Sonora y Tamaulipas. En el caso de Nuevo León se presentan también los resultados del único estudio cualitativo sobre descentralización a nivel jurisdiccional que se ha hecho en México. Los primeros cinco estados se descentralizaron en la primera etapa y firmaron otro convenio de descentralización en la segunda, mientras que Tamaulipas firmó sólo el segundo convenio.

\section{Descripción de los estados}

Sonora, Nuevo León y Tamaulipas están en la frontera con los Estados Unidos de América, y junto con Baja California Sur (BCS) tienen un ingreso per cápita, un porcentaje de población asegurada $(60-65 \%)$ y un índice de salud superior a la media nacional.

\section{Cuadro I \\ Supuestas Ventajas de LA DESCENTRALIZACIÓN ${ }^{17-20}$}

I. Fortalece la participación comunitaria y...

Cuando la comunidad participa en la planificación de los servicios y en la toma de decisiones, los servicios responden mejor a las necesidades locales y mejora su utilización.

Mejora el control de los recursos porque la comunidad puede exigir que se presten los servicios que son necesarios de forma oportuna.

2. Promueve la democracia

Al aumentar la participación de la comunidad en la planificación y gestión de servicios de salud se promueve la democracia.

3. Mejora la eficiencia

Los administradores organizan los servicios de acuerdo con las necesidades locales.

La comunidad conoce mejor los recursos disponibles y saben cómo maximizarlos.

La comunicación entre los niveles administrativos estatales, jurisdiccionales y municipales es más fácil y se evitan malentendidos y mal uso de recursos.

La toma de decisiones es más ágil cuando se hace localmente.

Se rompe con la cultura de las grandes burocracias y de los sindicatos.

4. Mejora la calidad de la atención

La población tiene más acceso a los gestores del sistema y exige mejores servicios.

Los trabajadores tienen mayor flexibilidad para organizar su jornada laboral.

Es más fácil establecer sistemas oportunos de suministros de insumos y medicamentos. 
En Colima, el ingreso per cápita y los índices de salud (mortalidad infantil y materna, esperanza de vida, y mortalidad general) son mejores que la media nacional, aunque $48 \%$ de la población es pobre y la mayoría $(52 \%)$ no tiene seguridad social. Guanajuato es un estado pobre; sólo 36\% de la población tiene seguridad social, y aunque su densidad de población es el triple de la media nacional, una tercera parte vive en zonas rurales dispersas. Sus indicadores de salud están por debajo de la media nacional.

Políticamente, los seis estados son muy diferentes: Tamaulipas y Colima siempre han sido gobernados por el Partido Revolucionario Institucional (PRI). En Guanajuato gobierna el Partido Acción Nacional (PAN) desde 1994; en BCS, una alianza del Partido de la Revolución Democrática (PRD) y el Partido del Trabajo (PT) han gobernado desde 1999. En Nuevo León ha habido una alternancia PRI-PAN en el gobierno desde la década de los noventa.

\section{Material y métodos}

El detalle de la metodología de cada uno de los estudios de caso puede consultarse en sus publicaciones. ${ }^{21-25}$ Basta decir que todos ellos fueron realizados entre 1998 y 2002 por diferentes investigadores, con metodologías cualitativas (entrevistas de profundidad, observaciones), que combinaron con datos de archivos (documentos legales y normativos), y datos cuantitativos publicados por las secretarías de salud federal y estatales (información financiera, recursos y desempeño del sistema, estadísticas de salud). En Guanajuato también se realizaron grupos focales. La semejanza metodológica, así como la coincidencia en los cargos de las personas entrevistadas (secretarios y otro personal administrativo y clínico de las secretarías estatales de salud; representantes de asociaciones profesionales, partidos políticos, miembros de las asambleas legislativas estatales, sindicatos de la salud y sociedad civil; académicos; periodistas; alcaldes y directivos del Instituto Mexicano del Seguro Social [IMSS], y del Instituto de Seguridad y Servicios Sociales de los Trabajadores del Estado [ISSSTE]) han permitido reunir los estudios en un solo artículo. En los seis estados se realizaron un total de 232 entrevistas de profundidad.

En Baja California Sur se efectuaron 27 entrevistas de profundidad, 15 de ellas con personas directamente involucradas en el proceso de descentralización: el secretario de Salud, los directores de Planificación, Regulación, Recursos Humanos, Administración, Servicios de Salud, directores de hospitales, jefes de jurisdicción, representantes de ONG y de sindicatos. ${ }^{21}$

En Guanajuato se hicieron un total de 30 entrevistas semiestructuradas a administradores de la Secretaría de
Salud, del IMSS, del ISSSTE, representantes de partidos políticos, sindicatos, académicos, ONG y periodistas. En una segunda etapa se realizaron 57 entrevistas con profesionales de la salud (médicos, enfermeras, trabajadores sociales y personal de laboratorio). ${ }^{22}$

En Sonora se llevaron a cabo 23 entrevistas de profundidad para estudiar el impacto de la descentralización en los servicios de salud estatales. Se entrevistó a administradores de la Secretaría de Salud, líderes de los partidos políticos y de sindicatos de trabajadores, personal de los institutos de seguridad social, miembros de la comisión de salud y académicos. ${ }^{23}$

En Colima se entrevistó a 20 personas, de las cuales 10 eran funcionarios de salud que habían participado en el proceso de descentralización o que habían presenciado su implementación. Otros entrevistados eran informantes calificados de la sociedad civil. ${ }^{24}$

En Tamaulipas se entrevistó a 38 personas, incluyendo jefes de división de la Secretaría de Salud, directores de hospitales, directivos de asociaciones profesionales, miembros de diversas ONG, directivos de partidos políticos y sindicatos, administradores del IMSS y académicos. ${ }^{25}$

El perfil de los 39 entrevistados en Nuevo León es igual al de Tamaulipas. ${ }^{25}$ Además se hicieron dos entrevistas de dos horas cada una al secretario de Salud y 18 entrevistas en las jurisdicciones.

Las entrevistas en Baja California Sur, Colima, Guanajuato y Sonora se grabaron y se transcribieron; en Nuevo León y Tamaulipas dos entrevistadores participaron en cada una de las entrevistas; uno de ellos tomaba notas que se transcribieron y consensuaron al terminar cada entrevista.

Para mantener el anonimato de los entrevistados, los identificamos por el cargo que ocupaban (jefe de servicios, al to cargo administrativo, etc.) y a veces cambiamos su género.

\section{Estudios de caso}

Utilizamos información proveniente de los estudios de $\operatorname{caso}^{21-25}$ para determinar si la segunda fase de la descentralización en los seis estados ha alcanzado los resultados que se presentan en el cuadro I. El análisis se basa en las conclusiones que aportaron los seis estudios.

\section{Fortalece la participación comunitaria y promueve} la democracia

En ninguno de los seis estados participaron los empleados de salud o las comunidades en la decisión de descentralizar ni en definir las características de la 
descentralización. En Colima, un funcionario de salud de alto nivel comentó:

"Fue una imposición de lo que teníamos que hacer; también tengo entendido que los gobernadores cuando iban a suscribir el acuerdo tenían serias dudas, inquietudes, pero finalmente hubo presiones para que lo firmaran."

El poder decisorio que se transfirió a los estados quedó centralizado en las secretarías estatales de salud. En Colima, otro funcionario que observó de cerca la descentralización dijo:

“Durante esa administración, todas las decisiones se realizaron en la cúpula del estado, no nos tomaron opinión..."

La participación de la sociedad civil ha sido mínima en los seis estados. Dirigentes de ONG de BCS hicieron los siguientes comentarios:

"La Secretaría de Salud a nivel federal a veces invita a nuestros representantes, pero aquí no. Como organización no nos toman en cuenta..."

"[Las organizaciones civiles] participan en lo que las autoridades quieren que participen, por ejemplo en comités de salud... pero alguien ha preparado la reunión y se aseguran de que la gente diga lo que los organizadores quieren."

Un dirigente sindical de Colima explicó:

“Únicamente nos dijeron de manera verbal sobre la reforma, nada oficial... no les preguntaron a los trabajadores..."

En Nuevo León, directivos de la secretaría estatal admitían abiertamente que la comunidad no debía participar en la planificación de los servicios. En BCS se crearon comités de salud en las escuelas y centros de salud, se hicieron foros en las comunidades, y en los centros de salud y hospitales se colocaron buzones de sugerencias; pero según un entrevistado, la información recabada no se utilizaba para la toma de decisiones. Un entrevistado comentó: "Yo veo a la sociedad muy apática... la gente hace lo que se le dice, no hay una discusión pública". Un representante de una ONG de Colima esclarecía la participación de la sociedad civil:

"A las reuniones que convocaba la Secretaría asistíamos diferentes instituciones, de salud y otras como nosotros, que tenemos actividades relacionadas con la salud; nos presentan un programa ya realizado y nos dicen dónde quieren que los apoyemos... nosotros no diseñamos el programa, son programas que vienen de México..."

En los estados no ha aumentado sensiblemente la transparencia de las secretarías de salud, que es uno de los pilares básicos de la democracia.

En BCS, el dirigente de una ONG comentó:

“... [las autoridades de salud] son muy renuentes a compartir información... no hay transparencia."

En el estado de Tamaulipas, un legislador de la oposición no pudo conseguir el presupuesto de la Secretaría Estatal de Salud, que debería ser accesible al público y ciertamente a los legisladores.

Una excepción fue Nuevo León, donde el secretario de Salud se propuso facilitar información y transfirió poder decisorio a las jurisdicciones.

\section{Participación a través del trabajo comunitario} y las cuotas de recuperación

En Sonora la participación consistía en el apoyo que la ciudadanía otorgaba durante las campañas de salud, si bien no se les incluía en el diseño ni se preguntaba qué tipo de servicios deseaban recibir. Los entrevistados denunciaron la tendencia de la secretaría a extraer dinero o trabajo gratuito de las comunidades más pobres: se esperaba que dedicasen tiempo a los aspectos logísticos de las campañas y dieran de comer a los trabajadores de salud que se trasladaban a las zonas rurales.

Para las secretarías de salud de BCS y de Guanajuato las cuotas de recuperación eran una forma de participación comunitaria, aunque ni en estos ni en ninguno de los otros cuatro estados se permitió que la sociedad civil participara en establecer su monto ni en definir su destino.

En Colima, donde una proporción elevada de la población sin seguridad social depende de los servicios de la Secretaría de Salud, los ingresos por cuotas siempre han sido superiores a la aportación del propio estado a los servicios de salud.

En Guanajuato se llegó a imponer cuotas de recuperación incluso en los servicios incluidos en el Programa Ampliado de Cobertura (PAC) financiado por el Banco Mundial, a pesar de que los servicios de este programa debían ser gratuitos.

En Tamaulipas las cuotas de recuperación por hospitalización en el Hospital General eran superiores a las de algunos hospitales privados en el vecino estado de Nuevo León, y ese hospital aumentó las cuotas de recuperación de la consulta externa sin consultar al 
Consejo Estatal de Salud. Aunque se violó la norma, nadie fue amonestado.

Las comunidades tampoco participaron al decidir el destino de los fondos recabados a través de las cuotas. Excepto en Tamaulipas, donde la comunidad consiguió que la legislatura obligara al Hospital General a perdonar las deudas adquiridas con el hospital a los 12 meses de haber recibido la alta hospitalaria, en ninguno de los estados se observó que los servicios de salud modificaran su funcionamiento en respuesta a las demandas de la sociedad civil o de los usuarios. En general, una parte de los ingresos por cuotas de recuperación se destinaba a la compra de medicamentos e insumos, pero también se utilizaban -como en el caso de Nuevo León-como incentivos para el personal, convirtiéndose en un impuesto regresivo. En Tamaulipas se utilizaban a discreción del director del establecimiento de salud donde eran recabadas.

\section{Mejora la eficiencia}

Los propulsores de la descentralización asumieron que al descentralizar la toma de decisiones los servicios responderían mejor a las necesidades de la comunidad, se maximizaría el uso de los recursos locales y aumentaría la eficiencia y productividad de los servicios. ${ }^{26}$

En ninguno de los seis estados se ha documentado un aumento de la eficiencia ya sea técnica o en la asignación, aplicación o distribución de los recursos (allocative efficiency) atribuible a la descentralización. En todos los estados, menos en BCS, aumentó la productividad (número de consultas por médico), pero no la eficiencia, porque en todos aumentaron los gastos, y se desconoce si mejoró o empeoró la calidad de los servicios.

Las entrevistas de profundidad sugieren que la allocative efficiency se deterioró: los nuevos vastos recursos se invirtieron preferencialmente en atención hospitalaria en vez de atención primaria, y en las zonas urbanas en lugar de las rurales. Un administrador de los servicios de salud de BCS comentó:

“Este gobierno ha destinado bastantes recursos a salud, pero es difícil ver para qué han servido... El hospital tenía un exceso de camas, y los administradores insistían en que todas tenían que estar ocupadas, y lo que es peor, querían abandonar el hospital y construir otro en la misma área, con un costo de 520 millones."

Otros dos administradores del mismo estado corroboraban:

"La influencia política es una mala cosa. Al final, los políticos ganan, las decisiones son básicamente políticas."
"¿Para qué sirve la descentralización si todo sigue funcionando igual?"

En Guanajuato, entre 1998 y 2000, más de 60\% de los fondos estatales para salud se invirtieron en hospitales de segundo y tercer nivel, aunque la atención primaria era prioritaria. Otros estados invirtieron en ésta pero algunos también fueron ineficientes. En Tamaulipas se siguieron construyendo centros de salud, aun sin tener el personal necesario para dar los servicios. Lo mismo sucedió en BCS, como sugiere el comentario de un jefe de servicios:

"[La descentralización] no ha aportado grandes beneficios. El centro de salud estaba bonito, pero vacío. Siguen teniendo los mismos problemas."

La pérdida de las economías de escala también contribuyó a disminuir la eficiencia del sector. Con la descentralización los estados se hicieron cargo de la compra de insumos y medicamentos a un precio superior al que anteriormente obtenían del nivel federal.

En Nuevo León no se había calculado el costo adicional que la compra fragmentada de medicamentos e insumos por las jurisdicciones podría representar. Por otra parte, $40 \%$ de los medicamentos se compraban en las farmacias privadas a precio de venta al público. Los otros estados tampoco habían calculado el costo adicional o la reducción de gasto originado por la compra directa de insumos y medicamentos.

En Colima se detectó otro uso ineficiente de recursos. El proceso incompleto de descentralización hizo que se siguieran implementando programas verticales que las secretarías estatales no podían alterar. Un informante en este estado explicó:

“Existen dos programas sin dirección... el de defectos del tubo neural; no tenemos un solo caso desde hace dos años, pero tenemos que seguir mandando informes y consumir recursos humanos y financieros..."

Para mejorar la eficiencia, a través de generar demanda, las secretarías de salud de Guanajuato y Nuevo León establecieron contratos y mejoraron los mecanismos de coordinación interinstitucional. Según un empleado de la Secretaría de Salud de Guanajuato:

"San Luis La Paz es un ejemplo de verdadera coordinación con el IMSS... encontramos una fórmula que en lugar de beneficiar a 120000 personas ahora beneficia a más de 300 000, y en lugar de construir otro hospital ahora utilizamos las camas del IMSS." 
En Nuevo León, la Secretaría de Salud firmó contratos con 20 municipalidades para mejorar la oferta de servicios. Los municipios también aportaron fondos. El secretario de Salud se propuso fortalecer los vínculos con los sistemas de seguridad social para ofrecer servicios a población asegurada en áreas rurales.

Por otro lado, se debe reconocer que la descentralización no es un requisito para establecer acuerdos interinstitucionales dentro de los estados. En Tamaulipas, antes de la descentralización los hospitales públicos eran autónomos y durante años tuvieron contratos con el IMSS y con el ISSSTE. Curiosamente, con la descentralización los hospitales perdieron su autonomía y pasaron a depender administrativamente de la Secretaría de Salud del estado.

Nuestro estudio no pretende cuantificar los cambios en la eficiencia producidos por la descentralización, sino identificar las causas que han podido contribuir o impedir su aumento. Según estándares internacionales el recurso médico sigue teniendo una productividad muy baja y la descentralización no ha aumentado el uso eficiente de este recurso.

Las influencias políticas -y en México también los sindicatos-, tienen en todos los países un impacto significativo en el uso de recursos. Es difícil establecer si después de la descentralización las influencias políticas estatales tienen un impacto más o menos negativo en la eficiencia, pero sí se puede afirmar que decisiones basadas en criterios políticos siguen entorpeciendo la implementación de mejoras en allocative efficiency.

La descentralización ha permitido innovaciones y aumentado la demanda y utilización de algunos servicios. Pero si se considera el aumento del gasto, los comentarios de los entrevistados en los seis estados, la construcción de clínicas y hospitales innecesarios, y los pocos cambios descritos, se concluye que la descentralización no ha mejorado la eficiencia en el uso de los recursos. Incluso podría insinuarse que ha habido un deterioro.

\section{Mejora la calidad de la atención}

Los estudios de calidad utilizan indicadores que miden más bien la calidez (satisfacción de los usuarios con los servicios) y la disponibilidad de insumos y medicamentos. La satisfacción de los usuarios con los servicios puede responder a la percepción de calidad de los mismos, pero también puede ser resultado de un trato atento, instalaciones agradables, cortos tiempos de espera, etc. Asimismo, las mejoras o deterioros de la morbimortalidad de la población responden a causas múltiples y las mejoras de los servicios de salud tienen un impacto reducido en esos cambios.
Se pueden formular hipótesis que correlacionan la descentralización y la mejora de la calidad a través de terceras variables (intervening variables). Todas las variables terceras tienen que ser calificadas. Por ejemplo, el acceso oportuno a medicamentos es una condición en muchos casos necesaria para mejorar la calidad, siempre que sean usados adecuadamente. De lo contrario, la presencia de medicamentos puede tener el efecto opuesto..$^{27-29}$

Las entrevistas indican que la mejora en la disponibilidad de equipos e insumos no fue uniforme en todos los estados. El estudio de Guanajuato concluía:

"Está claro que después de varios años de descentralización, importantes aspectos que afectan negativamente la calidad no han sido resueltos. Entre ellos se incluyen problemas relacionados con la carencia y calidad de los equipos, la calidad de la supervisión, el sistema de referencias, la carencia de medicamentos y la continuidad de atención médica."

En Colima, un informante que ocupaba un alto cargo en la administración afirmaba:

“La experiencia del año pasado fue gravísima, porque se retuvo el presupuesto durante cinco meses... eso afectó sobre todo a los medicamentos y [a la] continuidad en los programas... nunca respetan lo que solicitamos para medicamentos e insumos... existe deficiencia en el abasto de medicamentos y esto ocasiona el abandono del tratamiento o que [el paciente] no regrese más." [ ].

Varios de los encuestados en BCS indicaron que la falta de presupuesto para medicamentos y suministros empeoró la calidad de los servicios. Por el contrario, en Nuevo León mejoró notablemente la disponibilidad de medicamentos, pero no se realizaron estudios para determinar si también había mejorado su uso adecuado.

La descentralización de servicios de salud es una reforma técnica y administrativamente compleja, que exige el desarrollo de normas claras que se puedan implementar durante el proceso de cambio. Si se prepara una normativa adecuada y un sistema de supervisión efectivo se podría esperar que, manteniéndose todas las otras variables constantes, mejorase la calidad de los servicios de salud.

El personal entrevistado de la Secretaría de Salud de BCS se quejaba de la falta de supervisión. Los dirigentes de la Secretaría de Salud de Colima atribuían los problemas en el desempeño de los servicios a la falta de normatividad. En cambio, ante la carencia de una normativa, la Secretaría de Salud de Nuevo León decidió desarrollar sus propias normas. 
El hecho de que los estados interpretasen en forma marcadamente diferente el proceso de implementación de la descentralización sugiere una falta de planificación o de comunicación. Las encuestas no permiten afirmar que la calidad de la atención hubiera mejorado en cinco de los estados y tampoco permiten afirmar categóricamente que ésta hubiese mejorado en Nuevo León.

\section{Descentralización y equidad}

Si bien se sugirió, aunque no de forma taxativa, que la descentralización incrementaría la equidad, estudios realizados diez años después sugieren lo opuesto; ${ }^{30}$ entre otras razones porque las regiones más pobres tienen mayores dificultades para captar recursos humanos y financieros. Durante los estudios de caso no se recabó información específica para caracterizar el impacto de la descentralización en la equidad. Sin embargo, la dependencia de las cuotas de recuperación y el hecho de que algunos estados utilizaran su poder decisorio para incrementarlas sugiere que la descentralización pudo haber acrecentado la inequidad. Asimismo, el poco impacto del Fondo de Solidaridad Nacional de Salud añade peso a esta hipótesis.

El problema de establecer una cuota de solidaridad lo expresa bien el entonces secretario de Salud de Nuevo León, al decir que no le parecía correcto que Nuevo León pagara una cuota de solidaridad a la Secretaría de Salud para que fuese transferida a otros estados más pobres, ya que en su opinión la desorganización de los programas en estos estados era el resultado de la incompetencia de los políticos. Con todo, la existencia del Fondo no impidió que la inmensa mayoría de los fondos del gobierno central se siguieran adjudicando con base en presupuestos históricos y que los estados más pobres siguieran recibiendo menos recursos per cápita que el resto.

La decisión de la Secretaría de Salud de Nuevo León de utilizar los fondos de generación propia (provenientes casi en su totalidad de las cuotas de recuperación) para estímulos al personal era una forma de subvencionar los salarios de la clase media a partir de las contribuciones de los más pobres del estado, que son los que utilizan los servicios de la Secretaría.

El secretario de Salud de Guanajuato, refiriéndose al cambio que la descentralización produjo en la distribución del presupuesto federal, comentó:

“[antes de la descentralización] la distribución del pastel nacional [presupuesto de salud] no ha obedecido a una simetría de igualdad entre regiones... ahora sí, [se va hacer] con un criterio de cómo dividir el pastel en 32 rebanadas... para combatir las asimetrías a nivel nacional... desgraciadamente es una fórmula que no se aplica sobre $100 \%$ del recurso".
En Guanajuato había una clara preferencia por invertir recursos de salud en hospitales y servicios de especialidad urbanos, accesibles a toda la población según los decisores, aunque está bien establecido que la gran mayoría de los residentes rurales tienen dificultades para acceder a dichos servicios.

\section{Descentralización en las jurisdicciones de salud en Nuevo León}

Nuevo León es el único estado que descentralizó sus ocho jurisdicciones. El área metropolitana de la ciudad de Monterrey está dividida en cuatro, dos de las cuales agrupan a varios municipios aledaños que incluyen pequeños centros urbanos y áreas rurales; las dos jurisdicciones que sólo incluyen barrios tienen un poder decisorio limitado, ya que el municipio tiene recursos propios de salud y goza de cierta autonomía frente al estado. La jurisdicción número ocho cubre un área rural muy pobre, comparada con las otras, y depende totalmente de los servicios de salud estatales.

Los datos que presentamos provienen de documentos de la Secretaría de Salud estatal y de visitas de campo a cuatro jurisdicciones (dos urbanas y dos rurales) en las que se efectuaron varias entrevistas. ${ }^{31}$

Las jurisdicciones son responsables de los servicios de atención primaria y de las actividades de salud pública. Los hospitales responden directamente a la Secretaría Estatal de Salud. Hay dos tipos de centros de salud: centros básicos (con médicos generales y enfermeras, generalmente cumpliendo su año de servicio social obligatorio), y aquellos que ofrecen los cuatro servicios básicos de especialidad médica (medicina interna, pediatría, obstetricia/ginecología y cirugía), ubicados en centros urbanos.

\section{Organización de las jurisdicciones}

Cada jurisdicción tiene su propia estructura y sede central. El director es nombrado por el secretario de Salud estatal. Las jurisdicciones que visitamos tenían entre 200 y 500 empleados.

Las jurisdicciones constaban de divisiones (servicios de salud, epidemiología y medicina preventiva, regulación sanitaria, participación comunitaria, administración, educación, salud reproductiva, y estadística e información), y a su vez, algunas de estas divisiones tenían sus propias unidades.

\section{Transferencia de poder decisorio a las jurisdicciones}

La Secretaría de Salud de Nuevo León transfirió poder decisorio a las jurisdicciones al principio del gobierno. Las funciones que se descentralizaron fueron: 
- La organización administrativa de las oficinas jurisdiccionales.

- La negociación de la transferencia geográfica de personal dentro de las jurisdicciones, con la autorización previa del empleado y del Sindicato Nacional de Trabajadores de la Secretaría de Salud.

- La capacidad para captar recursos directos de los municipios.

- La administración del presupuesto (excepto salarios).

- El poder para establecer el monto de las cuotas de recuperación y las excepciones al pago de cuotas.

- Algunas funciones de regulación sanitaria.

Los directores de las jurisdicciones no hicieron cambios significativos en sus organigramas, en parte porque el control de personal seguía centralizado en la Secretaría de Salud federal. Por otra parte, la necesidad de transferir personal de una clínica a otra es importante porque la demanda de servicios puede cambiar con los cambios demográficos, con los contratos que las jurisdicciones podían firmar con los municipios o con los que la Secretaría estatal firmaba con los institutos de seguridad social.

Contratos con los municipios. A cambio de un pago por parte del municipio, la jurisdicción se comprometía a ofrecer a los trabajadores municipales servicios gratuitos, sin pago de cuotas de recuperación. Asimismo, se firmaron otros acuerdos más limitados; por ejemplo, un municipio ofreció un edificio para un programa de prevención de sida, y la jurisdicción se comprometió a buscar fondos para iniciar el programa. En otros municipios se compartieron gastos, como en la renovación de un centro de salud, la compra de un sistema de comunicación por radio, o la adquisición de una ambulancia.

La devolución de la capacidad decisoria a las jurisdicciones facilitó la capacidad de respuesta a las necesidades sanitarias percibidas por las autoridades municipales, si bien estas necesidades podrían responder a otros intereses; por ejemplo, para las autoridades municipales la compra de una ambulancia puede devengar dividendos políticos porque la población lo ve como una modernización de los servicios de salud.

Transferencia decisoria sobre recursos financieros. En 1997, la Secretaría de Salud permitió que las jurisdicciones utilizaran su presupuesto para comprar libremente todos los insumos que necesitaran a cualquier oferente, lo que hasta ese momento no se había dado en ningún otro estado de la federación.
Dos años después, en 1999, la Contraloría del estado comunicó a la Secretaría de Salud que la descentralización de compras a las jurisdicciones violaba la Ley Estatal de Adquisiciones, con lo que se dio marcha atrás al intento. A partir de esa decisión la compra directa por parte de las jurisdicciones se redujo a lo que podían adquirir con los recursos que generaban mediante las cuotas de recuperación. De acuerdo con el director de una jurisdicción:

“Desde 1999 hemos ido poco a poco perdiendo la capacidad de hacer compras directas con los fondos del estado..."

Un jefe de departamento de finanzas comentó:

"Tenemos que usar proveedores aprobados por el esta-
do... Algunas veces pagamos más de lo que debemos,
porque nosotros podemos encontrar proveedores que
nos ofrecen mejores precios..."

La Secretaría estatal sigue pagando directamente todos los costos fijos de las jurisdicciones, como agua, electricidad, teléfonos, mantenimiento de equipos, seguros, servicios de limpieza y las inversiones mayores de infraestructura.

Quizá el proceso más innovador que realizó la Secretaría de Salud fue la compra de medicamentos. Las jurisdicciones y los hospitales públicos hacían su pedido de medicamentos a la Secretaría de Salud estatal y ésta realizaba la licitación pública. Las empresas que ganaban los contratos se comprometían a aumentar o disminuir en $20 \%$ el pedido de medicamentos de cada jurisdicción y hospital sin modificar el precio unitario, lo que les permitía hacer ajustes de acuerdo con los cambios en la demanda de medicamentos. Además debían entregarlos directamente a los almacenes de las jurisdicciones, con lo cual fue posible cerrar el almacén estatal de medicamentos y reducir el tiempo de entrega. En caso de emergencia, las jurisdicciones y hospitales podían comprar los medicamentos directamente en las farmacias privadas.

En una entrevista un alto funcionario de la Secretaría de Salud de Nuevo León comentó:

\footnotetext{
"Ahorramos dinero al descentralizar el proceso de adquisición [de los medicamentos] a las jurisdicciones. Antes, los insumos y las medicinas no se distribuían cuando se necesitaban; los servicios y los usuarios se quejaban porque no tenían los insumos y las medicinas en el momento que se requerían... o porque los medicamentos que recibían tenían fechas de caducidad muy próximas. Ahora ya no tenemos ese problema."
} 
Un director de jurisdicción explicó:

"La descentralización nos ha permitido tener los medicamentos que necesitamos en 24 horas."

Las cuotas de recuperación. La Secretaría de Salud federal tenía normas sobre las cuotas de recuperación que la Secretaría de Salud de Nuevo León decidió no seguir. El secretario de Salud estatal comentó en una entrevista:

"Hay normas nacionales sobre la cantidad que podemos cobrar [por las cuotas de recuperación], pero nosotros no las seguimos. A final de cuentas, aquellos que pueden pagar pagan, y los que no pueden, no pagan."

Los directores de las jurisdicciones tenían clara esta postura y libertad para decidir las cuotas que querían cobrar en sus jurisdicciones. Uno de ellos indicó:

"Nuestra política es cobrar por todos los servicios y cobrar más de lo que la Secretaría de Salud federal indica... Es importante educar a los usuarios y a los médicos sobre la necesidad de pagar por los servicios... Cada centro de salud decide cuánto quiere cobrar, y yo apruebo las cuotas."

Para otro director las cuotas eran necesarias para ofrecer servicios de mejor calidad:

“Necesitamos las cuotas; sin ellas no se puede mantener el sistema. Ahora tenemos mejores servicios. No solamente podemos ofrecer mejor calidad de atención, sino que nuestra infraestructura física es también mejor."

Todos los entrevistados coincidieron en que nadie se quedaba sin atención médica por no poder pagar. Los directores de las jurisdicciones podían autorizar a los directores de las clínicas para que, de acuerdo con su propio criterio, no exigieran cuotas a pacientes de escasos recursos.

Las cuotas se convirtieron en uno de los pocos recursos que las jurisdicciones podían administrar libremente. Una buena parte de estos ingresos se asignaban a médicos y empleados administrativos de la Secretaría de Salud y jurisdicciones como premio por cumplir sus horarios de trabajo sin ausentismos laborales, es decir, por cumplir su contrato laboral. En estos casos, las cuotas se habían convertido en un impuesto claramente regresivo (transferencia de riqueza de personas de bajos ingresos a profesionales sanitarios).
Regulación sanitaria. Al principio de la descentralización la Secretaría de Salud estatal permitió que las jurisdicciones impusieran multas por infracciones de normas de higiene y saneamiento, pero esto se invalidó porque de acuerdo con la legislación estatal solamente la oficina jurídica de la Secretaría de Salud podía imponer multas, las cuales se pagaban a la Tesorería del estado. Eventualmente, la Tesorería devolvía los fondos recaudados a la Secretaría de Salud.

Antes de que se descubriera la ilegalidad de la transferencia, uno de los problemas fue prevenir la corrupción de los inspectores. Asimismo, aunque con la descentralización había aumentado el número de inspecciones, la falta de personal en la división de regulación sanitaria impedía que se realizaran todas las que eran necesarias.

\section{Discusión}

Los objetivos de los promotores de la descentralización no se alcanzaron en los estados estudiados. ${ }^{32-35}$ Con excepción de Nuevo León, donde se transfirió algún poder decisorio a las jurisdicciones, en los otros cinco estados el poder limitado que se transfirió de la Secretaría de Salud a los estados quedó concentrado en el gobierno estatal. Es necesario preguntarse cuáles han sido las causas del fracaso de la descentralización en México. Nuestro análisis nos permite sugerir lo siguiente:

1. La descentralización respondió a presiones del Banco Mundial y del FMI. Las políticas impuestas por agentes externos en respuesta a sus intereses tienen menos posibilidades de éxito si no coinciden con los intereses nacionales.

2. La descentralización fue impuesta desde el centro. Así, era difícil que prosperara.

3. El gobierno central no tuvo en cuenta la necesidad de capacitar al personal para asumir sus nuevas responsabilidades. De los seis estados solamente Nuevo León tenía personal capaz de reorganizar los servicios de salud, si bien cometieron errores al carecer de suficiente asesoría jurídica y esto fue costoso. Sin capacidad gerencial en los niveles inferiores, la descentralización tiene pocas posibilidades de éxito.

4. Antes de empezar la descentralización es necesario tener normas claras y específicas, y manuales operativos de las funciones que se van a descentralizar para cada nivel administrativo. La falta de normas para incorporar la participación comunitaria fue responsable de que cada estado definiera la 
participación comunitaria de formas diferentes, o simplemente no se aceptó que las comunidades participaran.

5. No se consideró el impacto de la descentralización en la equidad y no se promovieron políticas de distribución más equitativa de recursos y de protección de las poblaciones más vulnerables. En el nivel central y estatal es necesario que los presupuestos incluyan fondos de solidaridad para compensar la inequidad inherente al proceso descentralizador.

6. Es conveniente que una reforma profunda de los servicios de salud se consensúe con la oposición política y/o que se finalice, por lo menos en los aspectos más importantes, dentro del periodo de la administración que la propone. En México, una administración lanzó la reforma, fue paralizada por la siguiente, retomada por la que siguió, y olvidada más o menos definitivamente por las dos últimas; es decir, en México no se consiguió implementar la descentralización adecuadamente.

7. El presupuesto de salud que el gobierno central asignó a los estados aumentó considerablemente. La descentralización no consiguió sus objetivos y el Banco Mundial tampoco. Como documentó Wilkie desde hace 30 años, ${ }^{36}$ a lo largo del siglo 20 los estados y municipios mexicanos perdieron paulatinamente el derecho a recabar impuestos y su capacidad impositiva era muy limitada en un país federal. Por tanto, sin cambios legislativos que les permitieran cobrar impuestos no se podía esperar que reemplazaran los montos para salud cuando el gobierno central redujera sus asignaciones.

Asimismo, dado el reducido gasto público en salud en México antes de la descentralización -y los elevados gastos que ésta genera-se podría haber predicho que la reforma exigiría un aumento del gasto central.

\section{Conclusión}

La descentralización de servicios de salud es una política compleja. La decisión de llevarla a cabo debe responder a necesidades claramente definidas por la población y articuladas por sus representantes políticos. Se necesita un estudio minucioso de los obstáculos que pueden impedir su implementación, definir las políticas para minimizar la inequidad que necesariamente la acompaña, estudiar su costo y buscar los recursos adicionales necesarios.

Además es necesario hacer un estudio cuidadoso de las dimensiones legales y las soluciones a los problemas jurídicos que acompañan una descentralización. El entrenamiento de personal para las nuevas funciones que requiere el cambio, la estimación del personal adicional necesario y el destino del personal sobrante en el centro son prerrequisitos para potenciar el éxito. Todo esto es aún más necesario en los niveles jurisdiccional y municipal, donde la descentralización puede conseguir resultados más exitosos o convertirse en un fracaso más rotundo.

De los seis estados estudiados, solamente en Nuevo León se encontró que el personal administrativo de la Secretaría de Salud y de las jurisdicciones estaba satisfecho y optimista con el proceso descentralizador. Uno de seis es una proporción que deja pocas dudas sobre la percepción del resultado de la reforma.

Declaración de conflicto de intereses: Los autores declararon no tener conflicto de intereses.

\section{Referencias}

I. Peterson GE. Decentralization experience in Latin America: An overview of lessons and issues. LACDT Dissemination Note. Washington DC: The World Bank, 1994.

2. Litvack J, Ahmad J, Bird R. Rethinking decentralization in developing countries. Washington DC: The World Bank, 1998.

3. Banerji D. A simplistic approach to health policy analysis: the World Bank on the Indian health sector. Int J Health Serv 1994;24(I):I5I-I59.

4. Homedes N, Ugalde A. Twenty-five years of convoluted health reforms in Mexico. PLoS Med 2009;6(8):el000I24.

5. González-Block MA, Leyva R, Zapata O. Decentralizing health services in México: formulation, implementation, and results. Health Policy Plan 1989;4(4):30I-3I5.

6. Laurell AC. La reforma contra la salud y la seguridad social. México DF: Ediciones Era, 1997.

7. Gershberg Al. Decentralization and recentralization: Lessons from the social sectors in Mexico and Nicaragua. Washington DC: Inter-American Development Bank (Social Programs Division, Region II), 1998.

8. Birn AE. Federalist flirtations: the politics and execution of health services decentralization for the uninsured population in México: 19851995. J Public Health Policy 1999;20:8I-108.

9. Kumate J. Descentralización de los servicios de salud a población abierta. México DF: Secretaría de Salud, 1989.

10. Cardozo-Brum M. Análisis de la política descentralizadora del sector salud. México DF: Centro de Investigación y Docencia Económicas, 1993 II. Cardozo-Brum M. La descentralización de los servicios de salud en México: hacia la amnesia total o hacia la recuperación política. Gestión y Política Pública 1993:2:365-391.

12. Flamand Gómez L. Las perspectivas del nuevo federalismo: el sector salud. Las experiencias de Aguascalientes, Guanajuato y San Luis Potosí. México DF: Centro de Investigación y Docencia Económicas, 1998. 13. Instituto Nacional de Administración Pública (INAP). La descentralización de los servicios de salud: experiencias en los estados de Guanajuato, San Luis Potosí y Zacatecas. Guanajuato: Instituto de Administración Pública del Estado de Guanajuato, 2002.

14. Arrendondo A. El financiamiento a partir de la descentralización del sistema de salud en México: cambios, tendencias y evidencias, 1990-2000. Cuernavaca (México): Instituto Nacional de Salud Pública, 2002. 15. Magalhães Bosi ML, Mercado F, comp. Avaliação cualitativa de programas de saúde. Enfoques emergentes. Petrópolis: Editora Voces, 2006 
16. Magalhães Bosi ML, Mercado F, comp. Pesquisa cualitativa de serviços de saúde. Petrópoplis: Editora Voces, 2004.

17. Banco Mundial. Informe sobre el desarrollo mundial 1993. Invertir en salud. Washington DC: El Banco Mundial, 1993.

18. Ball G. Institutional and organizational development in decentralized projects. Washington DC: The World Bank, 1994.

19. Pan American Health Organization (PAHO). Developing and strengthening of local health systems in the transformation of national health systems. Document CD33/I4. Washington DC: PAHO, 1988.

20. Peterson GE. Decentralization in Latin America. World Bank Latin American and Caribbean Studies, Viewpoints. Washington DC: The World Bank, 1997.

21. Olvera-Santana L. Análisis de la implementación de la descentralización de los servicios de salud en el Estado de Baja California Sur 1996-2000 (tesis). Cuernavaca: Instituto Nacional de Salud Pública, 2002.

22. Arjonilla-Alday S. Políticas de ajuste macroeconómico, reforma del sector salud y el acceso, utilización y calidad de la atención en México. Informe Presentado al Instituto para el Desarrollo y la Investigación de Canadá. Cuernavaca: Instituto Nacional de Salud Pública de México, 2003. 23. Abrantes-Pêgo R. The slow and difficult institutionalization of health care reform in Sonora, 1982-2000. En: Homedes N, Ugalde A, ed. Decentralizing health services in Mexico. La Jolla: Center for U.S.-Mexican Studies, University of California-San Diego, 2006:187-208.

24. Maldonado-Hernández A. Análisis de la implementación de la descentralización de los servicios de salud para la población abierta en el estado de Colima (tesis). Cuernavaca: Instituto Nacional de Salud Pública, 2002.

25. Homedes N, Ugalde A. Nuevo León and Tamaulipas. Opening and closing a window of opportunity. En Homedes N, Ugalde A, ed. Decentralizing health services in Mexico. La Jolla: Center for U.S.-Mexican Studies, University of California-San Diego, 2006:23I-268.

26. Menéndez EL, comp. Participación social: metodología, problemas y expectativas. El caso Nicaragua. 1978-1989. México DF: Instituto Mora, 1999.
27. Reyes H, Guiscafré H, Muñoz O, Pérez-Cuevas R, Santoyo R, Gutiérrez $G$. Factores asociados a incumplimiento terapéutico y costo del desperdicio de antimicrobianos en infección respiratoria alta y diarrea aguda. Boletín Fármacos 1998; I (I):10-13

28. Gutiérrez G, Guiscafré H, Bronfman M, Walsh J, Martínez H, Muñoz $\mathrm{O}$. Changing physician prescribing patterns. Evaluation of an educational strategy for acute diarrhea in Mexico. Medical Care 1994;32(5):436-446. 29. Pérez-Cuevas R, Muñoz O, Guiscafré H. Patrones de prescripción terapéutica en infecciones respiratorias aguda y diarrea aguda en dos instituciones de Salud (IMSS, SS). IV. Características de la prescripción médica. Gaceta Médica Mexicana 1992;128:531-54I.

30. Saltam RB, Bankauskaite V, Vrangbæ K, ed. Decentralization in health care. Strategies and outcomes. Maidenhead (UK):Open University PressMcGraw-Hill, 2007.

3I. Homedes N, Ugalde A. Decentralization at the health district level. En Homedes N, Ugalde A, ed. Decentralizing health services in Mexico. La Jolla: Center for U.S.-Mexican Studies, University of California-San Diego, 2006:23I-268

32. Ase I. La descentralización de los servicios de salud en Córdoba. Salud Colectiva 2006;2(2):199-2/8.

33. De Groote T, De Paepe P, Unger JP. Colombia: in vivo test of health sector privatization in the developing world. Int J Health Serv 2005:35(I):I24-I4I.

34. Titelman D. Reformas al financiamiento del sistema de salud en Chile. Revista de la CEPAL, 1999;69:181-194.

35. Guevara EB, Mendias EP. A comparative analysis of the changes in nursing practice related to health sector reform in five countries of the Americas. Rev Panam Salud Publica 2002; 12(5):347-353.

36. Wilkie JW. The Mexican revolution: Federal expenditures and social change since 1910. Berkeley: The University of California Press, 1970. 